\title{
Evaluating drug interactions, adverse drug reactions, and level of adherence to highly active antiretroviral therapy regimen amongst HIV-positive patients who referred to an AIDS healthcare center in Fars, southern Iran: the first multifaceted study from Iran
}

\author{
Mojtaba Shafiekhani ${ }^{1}$, Shahram Karimi ${ }^{1}$, Mohammad ali Davarpanah ${ }^{2}$, Afsaneh Vazin ${ }^{1}$ \\ ${ }^{1}$ Department of Clinical Pharmacy, Faculty of Pharmacy, Shiraz University of Medical Sciences, Shiraz, Iran \\ ${ }^{2}$ HIV Research Centre, Shiraz University of Medical Sciences, Shiraz, Iran
}

\begin{abstract}
Introduction: To the best of our knowledge, this is the first multifaceted study from Iran that has evaluated the adverse drug reactions (ADR), drug-drug interactions (DDIs), and patient adherence to highly active antiretroviral therapy (HAART) regimen collectively. The HAART regimen is the most effective regimen in the treatment human immunodeficiency virus (HIV). However, this regimen is associated with ADRs and lack of adherence as well as DDIs.

Material and methods: This prospective cohort study was done from October 2014 to March 2015 on $200 \mathrm{HIV}$-positive patients who referred to an HIV/AIDS Research Centre receiving HAART regimen. DDIs was checked by lexi-com ${ }^{\circledR}$ software and Naranjo scale was used to evaluate the reported ADRs and then adherence of patients was evaluated by self-report.

Results: $96.50 \%$ of the patients reported at least one ADR. The central nervous system $(n=575$, $28.87 \%)$, gastrointestinal $(n=567,28.47 \%)$, and musculoskeletal adverse effects $(n=237,11.90 \%)$ were the most commonly reported. Overall, 302 DDIs, category C, D, and X, were recorded, of which, 259 interactions (85.8\%) were type C, 42 interactions (13.9\%) were type D, and only one interaction $(0.3 \%)$ was type X. 80 patients $(40 \%)$ had a history of discontinuation and did not use at least one dose of their medications. The main reasons for non-adherence to the regimen included: forgetfulness (43.75\%), unavailability of antiretroviral medications (23.75\%), and ADR (7.55\%).

Conclusions: This study showed a significant number of ADRs, DDIs, and nonadherence exist in our patients. It is clear that interventions for enhancing the ability of HIV-infected patients to cope with HAART regimens are warranted.
\end{abstract}

HIV AIDS Rev 2017; 16: 24-31 DOI: https://doi.org/10.5114/hivar.2017.65334

Key words: highly active antiretroviral therapy, adverse drug reaction, human immunodeficiency virus (HIV), adherence to ART, drug-drug interactions.

Address for correspondence: Associated Professor Afsaneh Vazin, Department of Clinical Pharmacy, Faculty of Pharmacy, Shiraz University of Medical Sciences, Zand Blvd, 7145654127, Shiraz, Iran, e-mail: vazeena@sums.ac.ir

\section{Article history:}

Received: 14.09.2016

Received in revised form: 11.11.2016

Accepted: 12.11.2016

Available online: 20.01.2017
International Journal of HIV-Related Problems

HIV \& AIDS

R e vi e w 


\section{Introduction}

Over the past two decades, human immunodeficiency virus (HIV) infection and acquired immunodeficiency syndrome (AIDS) has become a global epidemic, with political and economic consequences [1].

Based on the available data from the World Health Organisation (WHO), 35.5 million people are living with HIV, out of whom 1.6 million people died of AIDS-related diseases at the end of 2012 [2]. Based on the data of case registry system, a total of 30,727 people living with HIV had been identified in Iran by March 2015, and by September 2012, out of 28,000 Iranian HIV-positive patients eligible for antiretroviral therapy, only 3558 of them had received antiretroviral therapy [3].

In this regard, one of the most important factors in patients' non-adherence in taking these medications and discontinuation of highly active antiretroviral therapy (HAART) regimens is due to its adverse effects [4].

So far, more than $25 \%$ of patients discontinue treatment regimen during the first year, due to adverse drug reactions (ADRs) [5]. Antiretroviral therapy can cause a wide range of common ADRs, including gastrointestinal adverse effects such as: bloating, nausea, and diarrhoea, to more dangerous adverse effects such as anaemia, peripheral neuropathy, and hypersensitivity reactions [6]. Hepatotoxicity in about a third of patients with HIV occurs after starting HAART. In general, ADRs of HAART significantly affect the patient's quality of life and adherence to the treatment regimen [7]. However, the effectiveness of this treatment is directly related to the patients' adherence to the treatment regimen [7].

On the other hand, drug-drug interactions (DDIs) in HIV patients treated with HAART are important issues amongst these patients [8]. Drugs used to treat HIV are often susceptible to DDIs because many of them are metabolised via the CYP450 system, especially isoenzymes CYP3A4, CYP2D6, and CYP2C9/19, through which many medications are metabolised [9].

In spite the importance of this issue in HIV-positive patients, few studies have been conducted in Iran so far [2, 6]. Consequently, in this multifaceted study, the incidence of adverse effects of HAART, drug interactions, and also compliance of HIV-positive patients who referred to the Shiraz HIV/AIDS Research Centre were simultaneously investigated.

\section{Material and methods}

\section{Patient selection}

This prospective cohort study was carried out over a period of six months from October 2014 to March 2015 on 200 patients with AIDS who referred to Shiraz HIV/AIDS Research Centre affiliated with Shiraz University of Medical Sciences (SUMS), Shiraz, southern Iran.

In this centre, patients are treated free of charge by a general practitioner, infectious disease specialists, specialists in clinical psychology, and nurses.

After clinical and laboratory confirmation of HIV-infection, the patient is referred to this centre and uses the services, which includes monthly visits by an infectious disease specialist to determine the appropriate treatment regimen, follow-up, prescription of drugs for one month, as well as behavioural consultation.

In our study, inclusion criteria were as follows: (1) residency in Shiraz at the time of HIV diagnosis, (2) 18 years of age or older, (3) proven HIV-infection via western blot or HIV-1 RNA by PCR, and (4) receiving HAART. The patients were excluded if they did not refer to the centre for at least three months. The patients were followed up for six months. The study protocol was approved by the institutional review board and Medical Ethics Committee of the university and the patients were enrolled after obtaining written informed consent. It was explained that the results will remain confidential and they can withdraw from the study at any time.

\section{Data collection}

All the data were recorded by a pharmacist under the supervision of a clinical pharmacist and an infectious diseases specialist during the entire period of the study.

Data including sex, age, education level, marital status, employment status, duration after disease detection, interval between diagnosis and initiation of treatment, the drug reg-

Table 1. Lexi-Comp drug interaction software risk rating classifications for drug-drug interactions

\begin{tabular}{l|l|l}
\hline Risk rating & \multicolumn{1}{c}{ Description } & \multicolumn{1}{c}{ Action } \\
\hline A & $\begin{array}{l}\text { Data have not demonstrated either pharmacodynamic or pharmacokinetic interactions } \\
\text { between the specified agents }\end{array}$ & No interaction \\
\hline B & $\begin{array}{l}\text { Datta demonstrate that the specific agents may interact which each other, but there is } \\
\text { significant manner. The benefits of concomitant use of these two medications usually } \\
\text { outweigh the risk }\end{array}$ & No action needed \\
\hline C & $\begin{array}{l}\text { A patient-specific assessment must be conducted to determine whether the benefits } \\
\text { of concomitant therapy outweigh the risks }\end{array}$ & Modify regimen \\
\hline D & The risks associated with concomitant use of these agents usually outweigh the benefits & Avoid combination \\
\hline
\end{tabular}


Table 2. Characteristics of the patients enrolled in the study $(n=200)$

\begin{tabular}{|c|c|}
\hline Variables & $n(\%)$ \\
\hline \multicolumn{2}{|l|}{ Sex } \\
\hline Male & $124(62)$ \\
\hline Female & $76(38)$ \\
\hline \multicolumn{2}{|l|}{ Age } \\
\hline 20-29 years & $11(5.5)$ \\
\hline 30-49 years & $166(83)$ \\
\hline$>50$ years & $23(11.5)$ \\
\hline \multicolumn{2}{|l|}{ Education level } \\
\hline Illiterate & $12(6)$ \\
\hline Up to high school & $180(90)$ \\
\hline College education & $8(4)$ \\
\hline \multicolumn{2}{|l|}{ Marital status } \\
\hline Single & $43(21.5)$ \\
\hline Married & $101(50.5)$ \\
\hline Divorced & $33(16.5)$ \\
\hline \multicolumn{2}{|l|}{ Employment } \\
\hline Employed & $74(37)$ \\
\hline Unemployed & $126(63)$ \\
\hline \multicolumn{2}{|l|}{ Diagnosis time } \\
\hline Less than 1 year & $24(12)$ \\
\hline More than 1 year & $176(88)$ \\
\hline \multicolumn{2}{|c|}{ Duration of using medications } \\
\hline Less than 1 year & $91(45.5)$ \\
\hline More than 1 year & $109(54.5)$ \\
\hline \multicolumn{2}{|c|}{ Number of medications per day } \\
\hline Less than $10 /$ day & $160(80)$ \\
\hline More than $10 /$ day & $40(20)$ \\
\hline \multicolumn{2}{|l|}{ Comorbidities } \\
\hline Hepatitis C & $106(53)$ \\
\hline Hepatitis B & $11(5.5)$ \\
\hline Psychiatric diseases & $15(7.5)$ \\
\hline History of imprisonment & $80(40)$ \\
\hline Addiction & $11(5)$ \\
\hline Alcohol consumption & $9(4.5)$ \\
\hline
\end{tabular}

imen used, the number of pills consumed in a day, other underlying diseases, other medications, history of depression, alcohol consumption and substances abuse, and a history of imprisonment were recorded.

HAART adherence was assessed based on self-report during each visit, which has also been used in similar studies [10]. According to a previous study, patients with an adherence level of less than $95 \%$ were considered as non-adherent to HAART regimen [10].

If for any reason patients had forgotten, had adverse effects, imprisonment, finished tablets, bad taste of the drug, or any other reasons did not take his/her medication, it was recorded. Finally, the ADR of HAART, reported by patients or complications detected by the laboratory results were recorded.

Causality of any ADRs reported by patients were reviewed by Naranjo scale [11], and definite, probable, possible, or suspected adverse effects were determined. Hepatic laboratory abnormalities were considered as an increase of $10 \%$ in the mean plasma levels of hepatic enzymes (normal range: aspartate aminotransferase $[\mathrm{AST}] \leq 35 \mathrm{U} / \mathrm{l}$, alanine aminotransferase [ALT] $\leq 40 \mathrm{U} / \mathrm{l}$, alkaline phosphatase $[\mathrm{ALP}] \leq 110 \mathrm{U} / \mathrm{l}$, or bilirubin $\leq 22 \mu \mathrm{mol} / \mathrm{l}$ ) [12]. Acute kidney injury was defined as an abrupt (within 48 hours), absolute increase in creatinine serum concentration of $0.3 \mathrm{mg} / \mathrm{dl}(26.4 \mu \mathrm{mol} / \mathrm{l})$ from baseline: a percentage increase in creatinine serum concentration of $50 \%$ or oliguria of $0.5 \mathrm{ml} / \mathrm{kg}$ per hour for more than six hours [13].

Neutropaenia was defined as absolute neutrophil count (ANC) below 1500 cells/ $\mu l$, thrombocytopaenia was defined as platelet count $<100 \times 10^{3}$ platelets $/ \mathrm{mm}^{3}$, and anaemia was defined as haemoglobin $<13.5 \mathrm{~g} / \mathrm{dl}$ in males and haemoglobin $<12 \mathrm{~g} / \mathrm{dl}$ in females [14].

Drug-drug interactions were examined based on LexiInteract ${ }^{\mathrm{TM}}$ software, desktop version (Lexi-Comp Inc., 2008), and based on their importance were classified into five groups (A, B, C, D, X) (Table 1) and the severity of their clinical effects were determined [15].

\section{Statistical analysis}

The normality of the data distribution was checked using the Kolmogorov-Smirnov test. Continuous variables were presented as mean \pm SD. Categorical data were shown as percentages. Means of continuous variables were compared using Student's $t$-test. $\mathrm{X}^{2}$ was used to compare the association between categorical variables. In all statistical tests, a $p$ value $<0.05$ was considered as significant. All analyses were performed using SPPS, version 18.0 (SPSS, Inc., an IBM Company, Chicago, Illinois) statistical software.

\section{Results}

During the six months of the study 200 HIV-positive patients were enrolled. The mean \pm SD age of the patients was $39.5 \pm 7.3$ years with a range of 20 to 63 years. Demographic information of the patients is shown in Table 2.

The type and frequency of the prescribed HAART regimens for patients are listed in Table 3. The mean \pm SD of the number of drugs was $8.5 \pm 2.4$. The most common prophylactic medications was sulfamethoxazole-trimethoprim (43\%) followed by isoniazid (8\%) and azithromycin (7.5\%), respectively.

\section{Adverse drug reaction}

A total of 1991 ADR were recorded, and $96.50 \%$ of the patients reported at least one ADR. The mean \pm SD for the number of ADRs for each patient was $9.9 \pm 5.9$. Of the total 200 patients in our study, $77(38.50 \%)$ patients reported less than five ADRs, 59 (29.50\%) patients 6-10 ADRs, and 
64 patients (32\%) reported more than 11 ADRs. According to the Naranjo algorithm, all the ADRs reported by patients were possible and probable. The adverse effects of the central nervous system ( $n=575,28.8 \%)$, gastrointestinal system ( $n=567,28.4 \%)$, and musculoskeletal system $(n=237,11.9 \%)$ were the most commonly reported. Table 4 shows the details of detected ADRs.

HAART related hepatotoxicity was detected in our patients (Table 4). Elevated aminotransferase that reflect hepatocellular injury was the most common pattern of liver toxicity in our patients. Portal vein thrombosis was observed in one of our patients receiving didanosine as part of the therapy. In case of hepatotoxicity, treatment was discontinued and some modification was made to the HAART regimen.

Also, the total number of ADRs reported with triple therapy consisting of zidovudine, lamivudine, and efavirenz was higher than other drug regimens used. The mean \pm SD age of patients who reported at least one ADR was 39.6 \pm 7.4 years. Although the adverse effects of fatigue and hypertriglyceridaemia were reported more in patients under forty years old, using the Pearson correlation test a significant relationship was not observed between the number of reported ADRs and age $(p=0.38, R=-0.06)$. Also, between the number of medications and reported ADRs no significant correlation was observed ( $p=0.41, R=-0.05)$. Based on the $\chi^{2}$ test, by increasing the duration of drug use in patients, the risk of ADRs such as anaemia $(p=0.03)$, neutropaenia $(p=0.01)$, and rash $(p=0.01)$ significantly increased. However, there was no significant association between duration of drug use and the risk of other adverse effects.

ADRs such as vomiting $(p=0.02)$, rash $(p=0.02)$, dizziness $(p<0.001)$, anemia $(p<0.001)$, elevated triglycerides ( $p=0.008)$, and cholesterol $(p=0.009)$ were significantly higher in women than in men. However, other adverse effects such as increase in creatinine serum $(p=0.003)$, hyperbilirubinaemia ( $p=0.007)$, and increased aminotransferases $(p=0.01)$ were significantly higher in men than in women. In general, a significant association was not found between the number of adverse effects and patient gender $(p=0.14)$.

Moreover, there was no significant association between the number of adverse effects and underlying disease $(p=0.32)$.

\section{Drug interaction}

Overall, 302 drug interactions, category C, D, and X, were recorded, of which 259 drug interactions $(85.8 \%)$ were type $\mathrm{C}$, 42 interactions $(13.9 \%)$ were type $\mathrm{D}$, and one interaction $(0.3 \%)$ was type $\mathrm{X}$. One hundred and twenty-seven patients (63.5\%) had experienced at least one drug interaction (C, D, $\mathrm{X})$, mainly category $\mathrm{C}(62 \%)$. The most common interactions of group $\mathrm{C}$ were drug interactions between efavirenz-sulfamethoxazole-trimethoprim (31.66\%) and lamivudine-sulfamethoxazole-trimethoprim (31.66\%). The most common interactions in group D were drug interactions between efavirenz-methadone (59.52\%). The only drug interaction in cat-
Table 3. Type and frequency of prescribed highly active antiretroviral therapy regimen for the study population $(n=200)$

\begin{tabular}{|c|c|c|}
\hline Drug regimen & Frequency & Percentage \\
\hline Zidovudine, Lamivudine, Efavirenz & 150 & 75 \\
\hline Didanosine, Lamivudine, Efavirenz & 33 & 16.5 \\
\hline Tenofovir, Lamivudine, Efavirenz & 8 & 4 \\
\hline Abacavir, Lamivudine, Efavirenz & 4 & 2 \\
\hline Zidovudine, Lamivudine, Kaletra ${ }^{\circledR}$ & 2 & 1 \\
\hline Abacavir, Tenofovir, Kaletra ${ }^{\circledR}$ & 1 & 0.5 \\
\hline Tenofovir, Zidovudine, Efavirenz & 1 & 0.5 \\
\hline Abacavir, Didanosine, Kaletra ${ }^{\circledR}$ & 1 & 0.5 \\
\hline Total & 200 & 100 \\
\hline
\end{tabular}

egory X was the concurrent use of didanosine and ribavirin. The severity of interactions was mainly moderate (94.4\%) and only 5.6\% were major. According to the results of statistical tests, there was a significant association between the incidence of drug interactions and number of administered drugs $(p<0.001$ with interaction type $\mathrm{C}$ and $\mathrm{D})$, gender $(p=0.002$ with interaction type $C, p=0.009$ with interaction type $\mathrm{D}$ and $\mathrm{X}$ ), and underlying hepatic diseases ( $p=0.003$ with interaction type $\mathrm{C}, p<0.001$ with interaction type $\mathrm{D}$ and $\mathrm{X}$ ). Thus, the risk of drug interactions was significantly higher in men than in women, and higher in patients with underlying liver disease than in those without any hepatic disease.

\section{Adherence to antiretroviral therapy}

Eighty patients (40\%) had a history of discontinuation of at least one dose of their own medication. The range of duration of not taking medication was from one day to several months. In our study, the rate of non-adherence was $23.7 \%$. The main reasons for this include forgetfulness (43.7\%), unavailability of antiretroviral medications (23.7\%), and ADR (7.5\%).

The most important adverse events that led to non-compliance include: effects on the central nervous system (three cases), gastrointestinal system (one case), dermatological disorder (one case), and anaemia (one case).

The results showed that non-adherence to treatment in men was more frequent than in women. However, a significant association was not found between gender and adherence to treatment $(p=0.28)$. Also, there was no significant association between age and adherence to treatment ( $p=0.67)$, and there was no significant association between number of pills per day and adherence to treatment $(p=0.52)$. The lowest rate of non-compliance was reported amongst married patients (24\%) compared to singles (46.5\%) $(p=0.32)$. Also, a direct association was observed between level of education and lack of adherence. Thus, the highest non-compliance was reported in illiterate patients (50\%). 
Table 4. Details of the number and type of adverse drug reactions due to antiretroviral treatment regimen based on affected organ

\begin{tabular}{|c|c|c|c|}
\hline & Number & $\begin{array}{c}\text { Among } \\
\text { the total number } \\
\text { of complications (\%) }\end{array}$ & $\begin{array}{l}\text { Among the total } \\
\text { number of patients (\%) }\end{array}$ \\
\hline \multicolumn{4}{|l|}{ Gastrointestinal } \\
\hline Nausea & 104 & 5.2 & 52 \\
\hline Vomiting & 47 & 2.3 & 23.5 \\
\hline Diarrhoea & 34 & 1.6 & 17 \\
\hline Abdominal pain & 43 & 2.1 & 21.5 \\
\hline Anorexia & 96 & 4.8 & 48 \\
\hline Weight loss & 66 & 3.3 & 33 \\
\hline Bloating & 45 & 2.2 & 22.5 \\
\hline Bad taste & 80 & 4.0 & 40 \\
\hline Constipation & 26 & 1.3 & 13 \\
\hline Dyspepsia & 26 & 1.3 & 13 \\
\hline \multicolumn{4}{|l|}{ Dermatological } \\
\hline Rash & 60 & 3.0 & 30 \\
\hline Itching & 37 & 1.8 & 18.5 \\
\hline Urticaria & 52 & 2.6 & 26 \\
\hline \multicolumn{4}{|l|}{ Central nervous system } \\
\hline Headache & 48 & 2.4 & 24 \\
\hline Vertigo & 59 & 2.9 & 29.5 \\
\hline Anxiety & 31 & 1.5 & 15.5 \\
\hline Depression & 8 & 0.4 & 4 \\
\hline Insomnia & 28 & 1.4 & 14 \\
\hline Drowsy & 52 & 2.6 & 26 \\
\hline Nightmare & 65 & 3.2 & 32.5 \\
\hline Fatigue & 92 & 4.6 & 46 \\
\hline Agitation & 46 & 2.3 & 23 \\
\hline Difficult to concentrate & 71 & 3.5 & 35.5 \\
\hline Nervousness & 47 & 2.3 & 23.5 \\
\hline Hallucination & 28 & 1.4 & 14 \\
\hline \multicolumn{4}{|l|}{ Musculoskeletal } \\
\hline Myalgia & 56 & 2.8 & 28 \\
\hline Peripheral neuropathy & 80 & 4.0 & 40 \\
\hline Arthralgia & 31 & 1.5 & 15.5 \\
\hline Backache & 26 & 1.3 & 13 \\
\hline Weakness & 44 & 2.2 & 22 \\
\hline \multicolumn{4}{|l|}{ Cardiovascular } \\
\hline Chest pain & 5 & 0.2 & 2.5 \\
\hline \multicolumn{4}{|l|}{ Endocrine \& Metabolic } \\
\hline Increased FBS & 20 & 1.0 & 10 \\
\hline Increased TG & 23 & 1.1 & 11.5 \\
\hline Increased cholesterol & 19 & 0.9 & 9.5 \\
\hline
\end{tabular}


Table 4. Cont.

\begin{tabular}{|c|c|c|c|}
\hline & Number & $\begin{array}{c}\text { Among } \\
\text { the total number } \\
\text { of complications (\%) }\end{array}$ & $\begin{array}{l}\text { Among the total } \\
\text { number of patients (\%) }\end{array}$ \\
\hline \multicolumn{4}{|l|}{ Hematologic } \\
\hline Leukopaenia & 40 & 2.0 & 20 \\
\hline Neutropaenia & 31 & 1.5 & 15.5 \\
\hline Anaemia & 33 & 1.6 & 16.5 \\
\hline Thrombocytopaenia & 8 & 0.4 & 4 \\
\hline \multicolumn{4}{|l|}{ Hepatic } \\
\hline Increased AST & 34 & 1.7 & 17 \\
\hline Increased ALT & 46 & 2.3 & 23 \\
\hline Increased total bilirubin & 32 & 1.6 & 16 \\
\hline \multicolumn{4}{|l|}{ Genitourinary } \\
\hline Glycosuria & 2 & 0.1 & 1 \\
\hline Hematuria & 38 & 1.9 & 19 \\
\hline \multicolumn{4}{|l|}{ Renal } \\
\hline BUN increased & 6 & 0.3 & 3 \\
\hline Increased serum creatinine & 18 & 0.9 & 9 \\
\hline \multicolumn{4}{|l|}{ Others } \\
\hline Cough & 10 & 0.5 & 5 \\
\hline Sore throat & 3 & 0.1 & 1.5 \\
\hline Nasal congestion & 17 & 0.8 & 8.5 \\
\hline Sweating & 62 & 3.1 & 31 \\
\hline Total & 1991 & 100 & 200 \\
\hline
\end{tabular}

\section{Discussion}

During this study, about $96.5 \%$ of patients experienced at least one ADR, of which $2 \%$ discontinued their medications. In our study, most reported ADRs were related to the central nervous system and then the gastrointestinal system. In a cohort study by O'Brien et al., and also one by Khalili et al., gastrointestinal symptoms including nausea, vomiting, and diarrhoea were the most common reasons for discontinuation of treatment in the acute phase of HAART $[16,17]$. Perhaps one of the reasons for the insignificant differences between our results and those from Khalili et al. was that most of our patients used regimens containing efavirenz. In the study conducted by Khalili et al., carried out in 20052007, the most commonly prescribed regimens included zidovudine, lamivudine, and nelfinavir [16]. Due to poor compliance with nelfinavir as well as the availability of other drug choices in Iran, this drug was replaced with efavirenz. The most frequent HAART regimen used in our study population was zidovudine, lamivudine, and efavirenz. Efavirenz was usually associated with central nervous system toxicity, which has also been shown in several other studies [18-20].
Unlike the study by Mudzviti et al. [21] and the study by Lokhande et al. [22], in our study the most common adverse effect was not skin reaction. The reason for this could be lack of prescription of nevirapine in the treatment regimen of our patients.

The prevalence of severe hepatotoxicity with initiating HAART regimen was reported as $2-18 \%$ in some studies [23]. In our study, the rate of this complication was $23 \%$. The incidence of HAART hepatotoxicity depends on antiretroviral drug and host risk factors [23]. It seems that an increase in liver transaminases is a common adverse effect of lamivudine. This complication is also seen after consumption of zidovudine, didanosine, and efavirenz [24]. Regarding the host factors, severe hepatotoxicity is more frequent in HCV and/ or HBV coinfected individuals who have received HAART. This finding was also confirmed in the present study.

In our study there was also one case of portal thrombosis in a patient who was on didanosine for a long period of time. We postulate that this portal hypertension may be related to didanosine or HIV itself. However, implicating which medication might predispose patients to non-cirrhotic portal hypertension in patients with HIV is difficult. In the French case series and other case reports, exposure to didanosine 
was reported to be an important cause of vascular injury that led to non-cirrhotic portal hypertension [25-27].

In our study, unlike Khalili et al., simultaneous use of isoniazid was not a risk factor for liver toxicity $(p=0.25)$. This could be due to the fact that the number of patients taking isoniazid in our study was lower than that of Khalili's study (8\% vs. $35.5 \%)$ [16].

Moreover, metabolic complications such as dyslipidaemia were more common in patients treated with protease inhibitors. In the study of Bacchetti et al. carried out in 2005, dyslipidaemia was reported in about $50 \%$ of patients infected with HIV [28]. The lower frequency of this complication in our study $(11.5 \%$ increase in triglyceride and $9.5 \%$ increase in cholesterol), compared to other studies, may be due to not using protease inhibitors in our cohort.

More than half (63.5\%) of our study population were exposed to at least one drug interaction (C, D, X). In the study by Rajesh et al. [29], and Chaitanya et al. [30], from India, the prevalence of DDI in HIV-positive patients was $65.2 \%$ and $87.2 \%$, respectively. The variation in the frequency of DDIs in different studies can be due to the method of DDIs detection, screening, and different study settings.

Regarding the severity of interactions, in our study, the majority of drug interactions (89.8\%) were type C, $13.9 \%$ of interactions were type $\mathrm{D}$, and only one interaction $(0.3 \%)$ was type X. In the study by Marzolini et al., on the relationship between concomitant medications and risk of drug interactions in HIV-positive patients, it was concluded that the majority of drug interactions (59\%) were type C and only $2 \%$ were type $\mathrm{X}[31$ ], and with a sequence of severity of interaction similar to our results.

In our study, the most common interactions of D category were interactions between efavirenz and methadone, similar to the results obtained by Farhoudi et al. [2]. A retrospective study showed that starting efavirenz-containing regimens requires an increase in the dose of methadone to prevent withdrawal syndrome [32]. Nevertheless, in the current study, since most patients were receiving antiretroviral treatment for more than one year, they did not complain of any withdrawal syndrome.

In our study, there was a significant association between the occurrence of DDIs and the number of medications and also underlying disease and gender. The results were similar to those of Farhoudi et al. [2] except that the association between occurrence of DDIs and gender was not reported in the Farhoudi et al. [2] study.

Adherence has become one of the most important issues in suppressing HIV virus replication and avoiding resistance to antiretroviral drugs. In our study we used a self-reporting system to assess adherence to HAART regimen in patients, which validates this method that was used in other studies [33].

There is an acceptable correlation between self-reported drug adherence and HIV-1 plasma viral load, which validates self-reported drug intake in HIV-infected individuals taking HAART [36]. Developing treatment resistance can lead to increased hospitalisation rates, increased health care costs, reduced productivity, families and communities disorder, and increased morbidity and mortality.

In the study by Ioannidis et al. [34], the rate of non-adherence to HAART was reported between $15-37 \%$. In our study, the rate of non-adherence was $23.7 \%$. The mean rate of non-adherence in Khalili's study was $24.5 \%$ [10].

As mentioned earlier, forgetfulness and incomplete understanding of the medication benefits was one of the main issues for non-adherence to treatment [35], which is consistent with previous findings [36]. Also, in the study of Hosseini et al., some mentioned psychological reactions such as forgetfulness, hesitation, and exhaustion due to taking HAART create a great challenge for the participants' adherence to treatment [37].

Silva's study [38] showed that there was no significant relationship between adherence to treatment and age, gender, marital status, and education level. In our study, there was also no significant relationship between these factors and adherence to treatment. However, studies have shown that the number of tablets in the medication regimen is effective in adherence to the regimen [39]. However in our study, the number of pills per day did not show a significant association with adherence.

The present study has some limitations. First, detection of DDIs was only done according to one software package, while more DDIs might be found using other software packages such as Drug Interaction Fact ${ }^{\circledR}$. Second, we did not actively attempt to record over-the-counter medications, or traditional and herbal medicines; interactions may occur between these drugs and HAART regimens, which have been not reported so far. Another limitation of this study was that adherence was measured only by self-report, although this method is still one of the most important tools for measuring HARRT adherence.

\section{Conclusions}

This first multifaceted study in Iran showed that a significant number of ADRs and DDIs exist in patients receiving HAART regimens. A total of 1991 ADRs were recorded with adverse effects on the central nervous system, gastrointestinal system, and musculoskeletal system. Also, of the 200 patients, 127 had experienced at least one drug interaction. Eighty patients (40\%) had a history of discontinuation at least one dose of their own medications. The major cause that led to not taking at least one dose was forgetfulness. It is clear that interventions for enhancing the abilities of HIV-infected patients to cope with HAART regimens are warranted.

\section{Suggestion}

It seems that, to evaluate more accurately the level of non-adherence, other methods such as the pill counting method should also be used. OTC-medications and herbal drugs should be considered and evaluated for drug interactions. 


\section{Acknowledgement}

This research was performed by Shahram Karimi in partial fulfillment of the requirements for certification as a Pharm.D. in Pharmacy School at Shiraz University of Medical Sciences in Shiraz, Iran. The present paper was adopted from the proposal number "90-01-36-3865" approved by vice-chancellor for research affairs of Shiraz University of Medical Sciences.

\section{Conflict of interest}

The author's declared no potential conflicts of interest with respect to the research, authorship, and/or publication of this article.

\section{References}

1. Evans AS. Viral infections of humans: epidemiology and control. Springer Science \& Business Media, New York 2013.

2. Farhoudi M, Khalili H, Karimzadeh I, et al. Associated factors of drugdrug interactions of highly active antiretroviral therapy: report from a referral center. Expert Opin Drug Metab Toxicol 2015; 11: 471-479.

3. Joulaei H, Motazedian N. Primary health care strategic key to control HIV/AIDS in Iran. Iran J Public Health 2013; 42: 540-541.

4. Al-Dakkak I, Patel S, McCann E, et al. The impact of specific HIV treatment-related adverse events on adherence to antiretroviral therapy: a systematic review and meta-analysis. AIDS Care 2013; 25: 400-414.

5. Ortego C, Huedo-Medina TB, Llorca J, et al. Adherence to highly active antiretroviral therapy (HAART): a meta-analysis. AIDS Behav 2011; 15: 1381-1396.

6. Montessori V, Press N, Harris M, et al. Adverse effects of antiretroviral therapy for HIV infection. Can Med Assoc J 2004; 170: 229-238.

7. Chen WT, Shiu CS, Yang JP, et al. Antiretroviral Therapy (ART) side effect impacted on quality of life, and depressive symptomatology: a mixed-method study. J AIDS Clin Res 2013; 4: 218.

8. Nachega JB, Hsu AJ, Uthman OA, et al. Antiretroviral therapy adherence and drug-drug interactions in the aging HIV population. AIDS 2012; 26: S39-S53

9. de Maat MM, Ekhart GC, Huitema AD, et al. Drug interactions between antiretroviral drugs and comedicated agents. Clin Pharmacokinet 2003; 42: 223-282.

10. Khalili H, Rohani R, Seyedalinaghi S, et al. Adherence to antiretroviral therapy among Iranian HIV/AIDS patients. Curr Clin Pharmacol 2012; 7: 111-115.

11. Naranjo CA, Busto U, Sellers EM, et al. A method for estimating the probability of adverse drug reactions. Clin Pharmacol Ther 1981; 30: 239-245.

12. Ellis G, Goldberg D, Spooner R, et al. Serum enzyme tests in diseases of the liver and biliary tree. Am J Clin Pathol 1978; 70: 248-258

13. Thomas ME, Blaine C, Dawnay A, et al. The definition of acute kidney injury and its use in practice. Kidney Int 2015; 87: 62-73.

14. Boxer LA. How to approach neutropenia. Hematology Am Soc Hematol Educ Program 2012; 2012: 174-182.

15. Greene M, Steinman MA, McNicholl IR, et al. Polypharmacy, drug-drug interactions, and potentially inappropriate medications in older adults with human immunodeficiency virus infection. J Am Geriatr Soc 2014; 62: 447-453.

16. Khalili H, Dashti-Khavidaki S, Mohraz M, et al. Antiretroviral induced adverse drug reactions in Iranian human immunodeficiency virus positive patients. Pharmacoepidemiol Drug Saf 2009; 18: 848-857.

17. O'Brien ME, Clark RA, Besch CL, et al. Patterns and correlates of discontinuation of the initial HAART regimen in an urban outpatient cohort. J Acquir Immune Defic Syndr 2003; 34: 407-414.
18. Clifford DB, Evans S, Yang Y, et al. Long-term impact of efavirenz on neuropsychological performance and symptoms in HIV-infected individuals (ACTG 5097s). HIV Clin Trials 2009; 10: 343-355.

19. Ciccarelli N, Fabbiani M, Di Giambenedetto S, et al. Efavirenz associated with cognitive disorders in otherwise asymptomatic HIVinfected patients. Neurology 2011; 76: 1403-1409.

20. Simpson KN, Hanson KA, Harding G, et al. Review of the impact of NNRTI-based HIV treatment regimens on patient-reported disease burden. AIDS Care 2014; 26: 466-475.

21. Mudzviti T, Sibanda M, Gavi S, et al. Implementing a pharmacovigilance program to evaluate cutaneous adverse drug reactions in an antiretroviral access program. J Infect Dev Ctries 2012; 6: 806-808.

22. Lokhande AJ, Sutaria A, Shah BJ, et al. Changing incidence of nevirapine-induced cutaneous drug reactions: After revised guideline Nov 2011. Indian J Sex Transm Dis 2013; 34: 113-118.

23. Núnez M. Hepatotoxicity of antiretrovirals: incidence, mechanisms and management. J Hepatol 2006; 44: S132-S139.

24. Fenta DA, Hirigo AT. Changes in serum liver enzymes level after switching from stavudine/lamivudine to zidovudine/lamivudine in NNRTIs based anti-retroviral regimens in Hawassa, Southern Ethiopia. Am J Health Res 2014; 2: 397-403.

25. Carr A, Morey A, Mallon P, et al. Fatal portal hypertension, liver failure, and mitochondrial dysfunction after HIV-1 nucleoside analogue -induced hepatitis and lactic acidaemia. Lancet 2001; 357: 1412-1414.

26. Arey B, Markov M, Ravi J, et al. Nodular regenerative hyperplasia of liver as a consequence of ART. AIDS 2007; 21: 1066-1068.

27. Sandrine PF, Sylvie A, André E, et al. Nodular regenerative hyperplasia: a new serious antiretroviral drugs side effect? AIDS 2007; 21: 1498-1499.

28. Bacchetti P, Gripshover B, Grunfeld C, et al. Fat distribution in men with HIV infection. J Acquir Immune Defic Syndr 2005; 40: 121-131.

29. Rajesh R, Vidyasagar S, Varma DM, et al. Highly active antiretroviral therapy induced drug-drug interactions in Indian Human Immunodeficiency Virus positive patients. J Clin Med Res 2011; 3: 68-72.

30. Mastan S, Chaitanya G, Reddy KR, et al. Possible metabolic interactions between antiretroviral drugs and antidiabetic drugs: an overview. Int J Pharm Tech Res 2009; 1: 613-662.

31. Marzolini C, Elzi L, Gibbons S, et al. Prevalence of comedications and effect of potential drug-drug interactions in the Swiss HIV Cohort Study. Antivir Ther 2010; 15: 413-423.

32. Gourevitch MN, Friedland GH. Interactions between methadone and medications used to treat HIV infection. Mt Sinai J Med 2000; 69: 429-436.

33. Glass TR, De Geest S, Weber R, et al. Correlates of self-reported nonadherence to antiretroviral therapy in HIV-infected patients: the Swiss HIV Cohort Study. J Acquir Immune Defic Syndr 2006; 41: 385-392.

34. Ioannidis JP, Bassett R, Hughes MD, et al. Predictors and impact of patients lost to follow-up in a long-term randomized trial of immediate versus deferred antiretroviral treatment. J Acquir Immune Defic Syndr Hum Retrovirol 1997; 16: 22-30.

35. Li L, Lee SJ, Wen Y, et al. Antiretroviral therapy adherence among patients living with HIV/AIDS in Thailand. Nurs Health Sci 2010; 12: 212-220.

36. Shigdel R, Klouman E, Bhandari A, et al. Factors associated with adherence to antiretroviral therapy in HIV-infected patients in Kathmandu District, Nepal. HIV AIDS (Auckl) 2014; 6: 109-116.

37. Hosseini Z, Eftkhar H, Nedjat S, et al. Adherence to HIV/AIDS antiretroviral therapy among drug users: a qualitative study in Iran. Iran J Nurs Midwifery Res 2016; 21: 29-37.

38. da Silva RAR, Costa MMdN, Costa DARdS. Adherence to the antiretroviral treatment by people with aids: literature review. J Nurs UFPE online 2013; 7: 4227-4234.

39. Fairley C, Permana A, Read T. Long-term utility of measuring adherence by self-report compared with pharmacy record in a routine clinic setting. HIV Med 2005; 6: 366-369. 